\title{
Parent battering and the psychiatric and family correlates in children and adolescents
}

\author{
H. Perera ${ }^{1}$
}

Sri Lanka Journal of Child Health, 2006; 35: 128-32

(Key words: children, adolescents, violence to parents, obsessive compulsive disorder)

\begin{abstract}
The term "battered parents" was first used over two decades ago, but very few studies are available on the subject. This study examines an outpatient sample of children and adolescents where a key presenting feature was violent behaviour towards their parents. The commonest DSM IV axis I diagnosis was obsessive compulsive disorder with a prevalence of $25 \%$. The same diagnosis in the control group was $6.4 \%$. Anxiety and depressed mood dominated the clinical pictures in the study sample. Several crosscultural differences were apparent in age, behaviour and family context of the subjects when compared with the available studies. This study highlights the relevance of examining for presence of violence towards parents in children and adolescents who present with anxiety.
\end{abstract}

\section{Introduction}

Most scientific investigations on violent behaviour by children and adolescents toward other persons focus on bullying in schools, criminality and other antisocial conduct. Remarkably few studies have looked at violent behaviour towards their parents happening within their own homes. Gross under reporting and denial of such events by the parents could be a reason for this lack of information. One author describes this subject as "under-researched and a taboo subject associated with a sense of shame" . One of the earliest studies used the term "battered parents" and the phenomenon as a "new syndrome". The term "battering" is otherwise used where violence is adult led and the victims are children, spouses or the elderly. The study ${ }^{2}$ found that the victim most often was the mother. Also, the adolescents who have assaulted peers, siblings, teachers and strangers were more likely to threaten and inflict physical violence on the parents as well.

${ }^{1}$ Professor of Psychological Medicine, Faculty of Medicine, Colombo

(Received on 13 February 2006. Accepted on 20 April 2006)
The usual perpetrator was most often an economically dependent male aged 13 to 24 years $^{2}$. $18 \%$ of American children aged 3 to 17 years had carried out two or more violent acts on parents ${ }^{1}$. Oppositional behaviour, poor adaptability to stress, and low frustration tolerance are characteristics of youth who are aggressive to parents when compared with non aggressive controls ${ }^{3}$. At the same time, certain parent characteristics and parenting styles have been identified as risk factors that promote violence towards parents. Inconsistent parenting, unreasonable expectations, harsh and punitive discipline styles by parents are such characteristics ${ }^{1,4,5,6}$. Non-coping parents who inappropriately empower their children, also promote violence ${ }^{4}$.

Cross cultural studies show that Asian cultures report a relatively lower prevalence of aggressive behaviour in children when compared with non-Asian populations ${ }^{7,8}$. This difference has been explained on religious and cultural influences which discourage aggression and encourage self control, emotional restraint and social inhibition ${ }^{8}$. South Asian studies investigating violence towards parents by children and adolescents were not available. This study aims firstly, to investigate the prevalence of violent behaviour towards parents in a clinical sample of children and adolescents and secondly, to evaluate the associated psychiatric and family factors.

\section{Patients and Method}

The study sample was identified from children and adolescents who presented to outpatient clinics over a period of seven months. For this study "Parent Battering" was defined as parents being subjected to repeated physical violence by their child / adolescent offspring persisting for three months or more. The experience of violence was reported as markedly distressing and intimidating to at least one parent and given as a main reason for seeking medical help. Those children / adolescents, who were aggressive but not a primary concern of the parents, or where there was no physical violence towards the parents, were excluded from the study sample. 
Children / adolescents who fulfilled the working definition were selected for a more detailed assessment. All children / adolescents and parents were interviewed personally by the author. The clinical assessment and a semi-structured interview schedule (test-retest reliability $87.8 \%$ ) were used to gather information on behaviour, temperament and illness related data on the child / adolescent. Another semi-structured interview was used (test - retest reliability $83.3 \%$ ) to gather social and mental health data of parents, their perceived level of authority with the child / adolescent, their response to the violent behaviour and their beliefs about cause of violent behaviour.

All children / adolescents in the study sample were given a DSM IV Axis I diagnosis ${ }^{9}$. All other children / adolescents who presented for treatment to the same outpatient clinics and seen over the same study period, formed the control group. They too received DSM IV Axis I diagnoses. Ethical clearance was obtained from the Ethical Review Committee, Faculty of Medicine, Colombo.

\section{Results}

The study sample included 32 children and adolescents, aged 5 to 18 years. This study sample comprised $8.1 \%$ of the total patient number seen in the outpatient clinics for the study period. The control group had 393 subjects, aged 5-18 years.

Parents were targeted by all 32 children / adolescents. In $90.6 \%$ of cases, mother was the main target for assault. Both parents were equally targeted in $6.3 \%$ and father alone was the target only in one case (3.1\%). In $74.1 \%$ of cases, aggression towards any other persons was not reported. In $25.9 \%$, siblings were the target for violence. Grandparents and peers were also assaulted by $21.9 \%$. None attacked teachers or strangers.

Repeated hitting, mainly using fist was the commonest form of assault, often resulting in bruising. $31.3 \%$ used blunt objects to hit, with $12.5 \%$ using sharp objects as well. $56.3 \%$ also threatened the parents with death. One child stabbed her father with a kitchen knife causing a cut injury. An adolescent often held the mother down by her hair, until demands were met and also inflicted minor burns. Two mothers reported concussions following attacks on the head.

Anxiety was evident in 56.3\%. Further, 37.5\% had separation anxiety related to the parent at whom the aggression was mainly directed. A depressed mood was evident in $50 \%$ of cases. Obsessional thoughts with or without rituals were present in $53.1 \%$. Suicidal wishes were expressed by $25 \%$ but none had attempted deliberate self-harm. Disruptive behaviour at home was present in $62.5 \%$ and $15.6 \%$ had similar behaviour in school too. Destruction to property at home during outbursts of anger was reported in $37.5 \%$. Motor restlessness was reported in $56.3 \%$. Parents identified oppositional and defiant behaviour in $81.3 \%$. Comorbid features of conduct disorder were present in $31.3 \%$. Table I gives the primary diagnosis on DSM IV Axis I in the 32 subjects and the control group.

$53.1 \%$ were reported by the parents to have been demanding, disruptive and defiant even before the development of violent behaviour. 59.4\% were reported to have had low frustration tolerance. $34.4 \%$ have been timid and socially inhibited in school and other settings. $53.1 \%$ were oversensitive to criticism and easily became tearful. In $18.8 \%$ of cases, parents reported obedient and cooperative behaviour with them most of the time. $15.6 \%$ were also described as sensitive and conscientious about the parents.

In $84.4 \%$ of cases, both parents lived together with the child / adolescent. The rest lived with the mother, where parents had divorced / separated or the father worked abroad. In two cases, the mother left the family because of violence, but returned soon after. In $62.5 \%$ of cases, parents reported their marriage as good or satisfactory, and unsatisfactory or poor in $21.9 \%$. In $15.6 \%$ of cases, no comment was offered. $93.8 \%$ of the parents belonged to socio-economically middle class when their income and employment status were considered. In $18.8 \%$ of cases, parents felt that they had no authority over the offspring and in $50 \%$, the authority was present but inconsistent. The others had difficulty responding to this inquiry. 7 parents had a history of mental illness, some currently being on treatment for depression and schizophrenia. 5 fathers were abusing alcohol, three of whom showed violent behaviour at home.

In $40.6 \%$ cases, parents gave into the problem behaviour and in $34.4 \%$, help was requested from relatives and friends. No one was reported to the police. Parents gave physical punishments in 31.3\% and in 2 cases, the child was sent away to stay with relatives. Repeated warnings and taking away of privileges were imposed by parents in $9.4 \%$ cases.

In $62.5 \%$ of cases, the parents believed that the problem would worsen if restraints were imposed. The cause for violence was attributed to a mental 
illness in $43.8 \%$. Parents reported that $28.1 \%$ regretted violent behaviour later. A bad astrological period as the cause was considered only in one case.
A genetically related person was identified with the child / adolescent in character in $18.8 \%$.

Table 1

Comparison of DSM IV Axis I diagnosis of children /adolescents in study sample \& control group

\begin{tabular}{|l|c|c|}
\hline DSM IV Axis I Diagnosis & $\begin{array}{c}\text { Prevalence in study sample } \\
\mathbf{n = 3 2} \\
(\mathbf{M}: \mathbf{F} \mathbf{2 . 5} \mathbf{1})\end{array}$ & $\begin{array}{c}\text { Prevalence in control group } \\
\mathbf{n = 3 9 3} \\
(\mathbf{M}: \mathbf{F} \text { 2.3: 1) }\end{array}$ \\
\hline $\begin{array}{l}\text { Attention deficit /hyperactivity } \\
\text { disorder }\end{array}$ & $04(12.5 \%)$ & $66(13.8 \%)$ \\
\hline Obsessive-compulsive disorder & $08(25,0 \%)$ & $27(06.8 \%)$ \\
\hline $\begin{array}{l}\text { Schizophrenia and other } \\
\text { psychotic disorders }\end{array}$ & $01(03.1 \%)$ & $43(10.9 \%)$ \\
\hline Conduct disorder & $05(15.6 \%)$ & $09(02.3 \%)$ \\
\hline $\begin{array}{l}\text { Anxiety disorders (phobic } \\
\text { anxiety and separation anxiety } \\
\text { disorders) }\end{array}$ & $04(12.5 \%)$ & $55(14.0 \%)$ \\
\hline Tic disorder & $01(03.1 \%)$ & $11(02.8 \%)$ \\
\hline Oppositional defiant disorder & $02(06.2 \%)$ & 0 \\
\hline Cannabis abuse & $02(06.2 \%)$ & $02(0.5 \%)$ \\
\hline Mental retardation & 0 & $17(04.3 \%)$ \\
\hline Learning disorders & 0 & $86(21.9 \%)$ \\
\hline Asperger disorder & $02(06.2 \%)$ & 0 \\
\hline Bipolar disorder & 0 & $23(05.8 \%)$ \\
\hline Depressive disorder & 0 & $15(03.8 \%)$ \\
\hline Eating disorders & 0 & $05(01.3 \%)$ \\
\hline Dissociative disorder & 0 & $05(01.3 \%)$ \\
\hline Stuttering & 0 & $06(01.5 \%)$ \\
\hline No clear DSM IV diagnosis & $03(09.4 \%)$ & $23(05.8 \%)$ \\
\hline & $32(100 \%)$ & $393(100 \%)$ \\
\hline
\end{tabular}

\section{Discussion}

Similar to previous studies from other cultures, mother is the most affected parent and the majority comes from middle class families. However, differences are many. Firstly, $75 \%$ of our cases were below 15 years of age. Secondly, in nearly 3/4 of cases violence was confined to the home. Thirdly, over $1 / 3$ of individuals were reported as timid and socially inhibited outside home. Fourthly, symptoms of anxiety, depressed mood and obsessions dominated as presenting features and obsessive compulsive disorder (OCD) was the commonest single diagnosis. In contrast, commonest diagnosis identified with violent behaviour in other studies was conduct disorder (CD) with secondary diagnoses of developmental, neurological, emotional and behaviour disorders ${ }^{1,2,3}$. However, children / adolescents with OCD show aggressive behaviour to family members ${ }^{10,11,12}$ and this was reported also in an Asian study ${ }^{13}$. Parental over-involvement and disagreement with the OCD related behaviour is a trigger for aggression ${ }^{12,14,15}$. The prevalence of OCD in our study group becomes even more relevant when compared with the control group, where only $6.4 \%$ had this diagnosis. Also, CD was diagnosed only in $15.6 \%$ in the study sample, but was 7 times more prevalent than that in the control group. Further, opposition and defiance was reported in $81.3 \%$, though a diagnosis of CD could not be substantiated in many. Attention deficit hyperactivity disorder (ADHD), seizure disorders and brain damage that promote violence through low stress tolerance and poor impulse control did not display prominently in the study group. In contrast to other studies ${ }^{1,16,17}$, depressive disorder was not associated with violence in our sample, even though depressed mood was a frequent occurrence. In keeping with the finding that violence is infrequent in schizophrenia ${ }^{18}$, the prevalence of this disorder was low in the study 
sample. In contrast to known family factors that promote violence in children / adolescents 19,20,21, punitive parenting behaviour was not identified in our study.

\section{Clinical Relevance}

Possibility of violence to parents should be routinely considered in assessment of children and adolescents with OCD and other anxiety disorders. For effective clinical management, it is important to identify specific characteristics in the child and parents, including, beliefs and values in parents about violent behaviour.

\section{References}

1. Bailey S. Violent children: a frame work for assessment. Advances in Psychiatric Treatment 2002; 6: 97-106.

2. Harbin HT, Madden DJ. Battered parents, a new syndrome. American Journal of Psychiatry 1979; 136: $1288-91$.

3. Nock MK, Kazdin AE. Parent-directed physical aggression by clinic-referred youth. Journal of Clinical Child and Adolescent Psychology 2002; 31: 193-205.

4. Baden AD, Howe GW. Mothers' attribution and expectancies regarding their conduct disordered children. Journal of Abnormal Child Psychology 1992; 20: 467-85.

5. Harbin HT, Madden DJ. Assaultative adolescents: family decision making parameters. Family Process 1983; 22:109-18.

6. Snyder J, Schrepferman L, St. Peter C. Origins of antisocial behavior: negative reinforcement and affect dysregulation of behavior as socialization mechanisms in family interaction. Behavior Modification 1997: 21: 187- 215.

7. Weine AM, Phillips JS, Achenbach TM. Behavioural and emotional problems among Chinese and American children: parent and teacher reports for ages 6 to 13. Journal of Abnormal Child Psychology 1995: 23: 619-39.
8. Weisz JR, Suwanlert S, Chaiyasit W, Weiss B, Achenbach TM, Eastman KL. Behavioural and emotional problems among Thai and American adolescents: parent reports for ages 12 to 16 . Journal of Abnormal Psychology 1993; 102: 395-403.

9. Diagnostic and Statistical Manual of Mental Disorders, $4^{\text {th }}$ ed. Washington DC. American Psychiatric Association; 1994.

10. Riddle MA, Scahill L, King R, Hardin MT, Towbin KE, Ort SI, et al. Obsessive-compulsive disorder in child and adolescents: phenomenology and family history. Journal of the American Academy of Child and Adolescent Psychiatry 1990; 29: 766-72.

11. Stephen RJ, Sandor P. Aggressive behaviour in children with Tourette syndrome and comorbid attention deficit hyperactivity disorder and obsessive compulsive disorder. Canadian Journal of Psychiatry 1999; 44: 1036-42.

12. Calvocoressi L, Lewis B, Harris M, Trufan SJ, Goodman WK, McDougle CJ, et al. Family accommodation in obsessive-compulsive disorder. American Journal of Psychiatry 1995; 152: 441-3.

13. Honjo S, Hirano C, Murase S, Kaneko T, Sugiyama T, Ohtaka K, et al. Obsessivecompulsive symptom in childhood and adolescence. Acta Psychiatrica Scandinevica 1989, 80: 83-91.

14. Merkel WT, Pollard CA, Weiner RL, Stabler CR. Perceived parental characteristics of patients with OCD, depression and panic disorder. Child Psychiatry and Human Development 1993; 24: 49-57.

15. Cooper M. OCD: effect on family members American Journal of Orthopsychiatry 1996; 66: 296-304.

16. Goodyer IM, Herbert J, Secker SM. Short term outcome of major depression: 1. Comorbidity and severity at presentation as predictor of persistent disorder. Journal of the American Academy of Child and Adolescent Psychiatry 1997; 36:179-87. 
17. Knox M, King C, Hanna GL, Logan D, Ghaziuddin N. Aggressive behaviour in clinically depressed adolescents. Journal of the American Academy of Child and Adolescent Psychiatry 2000; 39: 611-8.

18. Clare P, Bailey S, Clark A. Relationship between psychotic disorders in adolescents and criminally violent behaviour. British Journal of Psychiatry 2000: 177: 275-9.

19. Clark A. Proposed treatment of adolescent psychosis: I. schizophrenia and schizophrenia like psychoses. Advances in Psychiatric Treatment 2001; 7:16-23.
20. Calhoun AD, Clark-Jones F. Theoretical frameworks - developmental psychopathology, the public health approach to violence, and the cycle of violence. Pediatric Clinics of North America 1998; 45: 281-91.

21. Edari R, McManus P. Risk and resiliency factors for violence. Pediatric Clinics of North America 1998; 45: 293-305.

22. Stormshak EA, Bierman KL, McMahon RJ, Lengua LJ. Parenting practices and child disruptive behaviour problems in early elementary school. Conduct Problem Prevention Research Group. Journal of Clinical Child Psychology 2000; 29: 17-29. 